\title{
REMARKS ON TWO RECENT RESULTS ABOUT POLYNOMIALS WITH PRESCRIBED ZEROS
}

\author{
M. A. QAZI
}

\begin{abstract}
We make some observations about the results contained in a paper published in this journal in the year 2011.
\end{abstract}

\section{INTRODUCTION}

For a polynomial $p(z)$, let $M(p, \rho):=\max _{|z|=\rho}|p(z)|$.

The following result was proved by Aziz [1].

Theorem A. If $p(z)$ is a polynomial of degree $n$, which does not vanish in $|z|<k, k \geq 1$, then

$$
M(p, R) \leq \frac{R^{n}+k^{n}}{1+k^{n}} M(p, 1) \text { for } R>k^{2},
$$

provided $\left|p^{\prime}\left(k^{2} z\right)\right|$ and $\left|p^{\prime}(z)\right|$ attain the maximum at the same point on $|z|=1$. The result is best possible with equality for $p(z)=z^{n}+k^{n}$.

The next result appears in [2].

Theorem B. If $p(z)$ is a polynomial of degree $n$, which does not vanish in $|z|<k, k<1$, then

$$
M(p, r) \geq \frac{r^{n}+k^{n}}{1+k^{n}} M(p, 1) \text { for } 0<k<r<1,
$$

provided $\left|p^{\prime}(z)\right|$ and $\left|q^{\prime}(z)\right|$ attain the maximum at the same point on $|z|=1$, where $q(z)=z^{n} \overline{p(1 / \bar{z})}$. The result is best possible and equality holds for $p(z)=z^{n}+k^{n}$.

In a paper published in this journal in the year 2011, which is quoted as item number [3] in the list of references, Dewan and Hans make the following statements (see Theorems 1 and 2 of [3]).

2010 Mathematics Subject Classification. 30A10, 30C10, 30C15.

Key words and phrases. Polynomials, maximum modulus, zeros, extremal problems. 
Theorem 1. If $p(z)=a_{0}+\sum_{\nu=\mu}^{n} a_{\nu} z^{\nu}, 1 \leq \mu<n$ is a polynomial of degree $n$, which does not vanish in $|z|<k, k \geq 1$, then

$$
M(p, R) \leq \frac{R^{n}+k^{n}\left(1+k^{n-\mu+1}\right)-k^{2 n}}{1+k^{n-\mu+1}} M(p, 1) \text { for } R>k^{2},
$$

provided $\left|p^{\prime}\left(k^{2} z\right)\right|$ and $\left|p^{\prime}(z)\right|$ attain the maximum at the same point on $|z|=1$.

Theorem 2. If $p(z)=a_{n} z^{n}+\sum_{\nu=\mu}^{n} a_{n-\mu} z^{n-\mu}, 1 \leq \mu<n$ is a polynomial of degree $n$, which does not vanish in $|z|<k, k<1$, then

$$
M(p, r) \geq\left(\frac{r^{n-\mu+1}+k^{n-\mu+1}}{\lambda^{n-\mu+1}+k^{n-\mu+1}}\right)^{\frac{n}{n-\mu+1}} M(p, \lambda) \text { for } 0<k<r<\lambda \leq 1,
$$

provided $\left|p^{\prime}(z)\right|$ and $\left|q^{\prime}(z)\right|$ attain maximum at the same point on $|z|=1$, where $q(z)=z^{n} \overline{p(1 / \bar{z})}$. The result is best possible and equality holds for $p(z)=\left(z^{n-\mu+1}+k^{n-\mu+1}\right)^{\frac{n}{n-\mu+1}}$.

\section{Some Remarks on Theorems 1 And 2}

Remark 1. Theorem 1 is much ado about nothing. In the case where $\mu=1$, Theorem 1 is the same as Theorem A. The authors acknowledge this in [3] (see Remark 1 on page 12). For $1<\mu<n$, their result is weaker than Theorem A which it is supposed to refine. The upper bound for $M(p, R)$ given in (1) is smaller than the one given in inequality (3). This can be seen as follows.

Pay attention to the fact that $k>1$ and $R>k^{2}$. Clearly,

if and only if

$$
\frac{R^{n}+k^{n}}{1+k^{n}}<\frac{R^{n}+k^{n}\left(1+k^{n-\mu+1}\right)-k^{2 n}}{1+k^{n-\mu+1}}
$$

$$
\left(R^{n}+k^{n}\right)\left(1+k^{n-\mu+1}\right)<\left(R^{n}+k^{n}\left(1+k^{n-\mu+1}\right)-k^{2 n}\right)\left(1+k^{n}\right)
$$

which holds if and only if

$k^{n}\left(1+k^{n-\mu+1}\right)-k^{n}\left(1+k^{n-\mu+1}\right)\left(1+k^{n}\right)+k^{2 n}\left(1+k^{n}\right)<R^{n}\left(k^{n}-k^{n-\mu+1}\right)$, i.e., if and only if

$$
-k^{2 n}\left(1+k^{n-\mu+1}\right)+k^{2 n}\left(1+k^{n}\right)<R^{n}\left(k^{n}-k^{n-\mu+1}\right),
$$

which in turn holds if and only if

$$
k^{2 n}\left(k^{n}-k^{n-\mu+1}\right)<R^{n}\left(k^{n}-k^{n-\mu+1}\right),
$$

i.e., if and only if $R>k^{2}$, being given that $k>1$. 
In [3], the authors consider what can be seen as a subclass of polynomials satisfying the conditions of Theorem A and then end up with a conclusion that is weaker than the one given in Theorem A.

Remark 2. Going through the proof of Theorem 2 we notice that it uses the following statement which the authors call Lemma 1 (see [3, page 13]).

Lemma 1. Let $p(z)=a_{n} z^{n}+\sum_{\nu=\mu}^{n} a_{n-\nu} z^{n-\nu}, 1 \leq \mu<n$ be a polynomial of degree $n$, having no zero in $|z|<k, k \leq 1$ and $q(z)=z^{n} \overline{p(1 / \bar{z})}$. If $\left|p^{\prime}(z)\right|$ and $\left|q^{\prime}(z)\right|$ attain maximums at the same point on $|z|=1$, then

$$
M\left(p^{\prime}, 1\right) \leq \frac{n}{1+k^{n-\mu+1}} M(p, 1) .
$$

This "so-called lemma" is invalid for $1<\mu<n$. In order to see this, consider the polynomial

$$
p(z)=p(\varepsilon, z)=z^{n}+\varepsilon z^{n-\mu}+\ell^{n},
$$

where $\ell \in(0,1)$ and $\varepsilon>0$. The zeros of a polynomial are continuous functions of the coefficients (see [4, p. 10, Theorem 1.3.1]). Hence, for small values of $\varepsilon$, the polynomial $p(\varepsilon, z)$ has no zeros in $|z|<k=k(\varepsilon)$ for some $k \in(0,1)$, where $k(\varepsilon) \rightarrow \ell$ as $\varepsilon \rightarrow 0$. Clearly,

$$
M(p, 1)=\max _{|z|=1}|p(z)|=1+\varepsilon+\ell^{n} \text { and } M\left(p^{\prime}, 1\right)=\max _{|z|=1}\left|p^{\prime}(z)\right|=n+(n-\mu) \varepsilon .
$$

Thus, if (5) was true, then we would have

$$
\frac{n+(n-\mu) \varepsilon}{1+\varepsilon+\ell^{n}} \leq \frac{n}{1+k(\varepsilon)^{n-\mu+1}}, \varepsilon>0 .
$$

Letting $\varepsilon \rightarrow 0$ in (6), we obtain

$$
\frac{n}{1+\ell^{n}} \leq \frac{n}{1+\ell^{n-\mu+1}}
$$

i.e., $1 \leq \ell^{\mu-1}$, which is a contradiction, since $\ell \in(0,1)$ and $1<\mu<n$ by hypothesis. Thus, Lemma 1 is invalid.

Since Lemma 1 is invalid, so is Theorem 2 because the proof of Theorem 2 depends on Lemma 1.

Remark 3. The last sentence in the statement of Theorem 2 is: "The result is best possible and equality holds for $p(z)=\left(z^{n-\mu+1}+k^{n-\mu+1}\right)^{\frac{n}{n-\mu+1}}$ ". The authors seem to believe that $p(z)=\left(z^{n-\mu+1}+k^{n-\mu+1}\right)^{\frac{n}{n-\mu+1}}$ is a polynomial. There is absolutely no mention of the fact that for $p(z)=$ $\left(z^{n-\mu+1}+k^{n-\mu+1}\right)^{\frac{n}{n-\mu+1}}$ to be a polynomial, $\frac{n}{n-\mu+1}$ must be an integer. 


\section{REFERENCES}

[1] A. Aziz, Growth of polynomials whose zeros are within or outside a circle, Bull. Austral. Math. Soc., 35 (1987), 247-256.

[2] K. K. Dewan and S. Hans, Growth of polynomials whose zeros are outside a circle, Ann. Univ. Mariae Curie-Sklodowska, Lublin-Polonia, LXII (2008), 61-65.

[3] K. K. Dewan and S. Hans, Growth of the maximum modulus of polynomials with prescribed zeros, Sarajevo J. Math., 7 (19) (2011), 11-17.

[4] Q. I. Rahman and G. Schmeisser, Analytic Theory of Polynomials, Clarendon Press. Oxford, 2002.

(Received: June 5, 2013)

Department of Mathematics

Tuskegee University

Tuskegee, AL 36088

U. S. A.

qazima@aol.com 